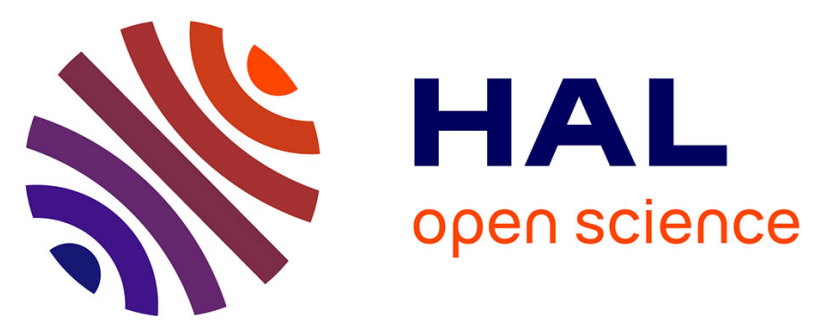

\title{
Numerical simulation of failure prediction for ceramic tools: comparison with forging test results
}

Frédéric Deschaux-Beaume, Fabrice Schmidt, Nicole Fréty, Thierry Cutard, Jean-Claude Boyer, Christophe Levaillant

\section{- To cite this version:}

Frédéric Deschaux-Beaume, Fabrice Schmidt, Nicole Fréty, Thierry Cutard, Jean-Claude Boyer, et al.. Numerical simulation of failure prediction for ceramic tools: comparison with forging test results. Journal of Materials Processing Technology, 1999, 92-93, pp.502-507. 10.1016/S0924-0136(99)002253. hal-02050668

\section{HAL Id: hal-02050668 https://imt-mines-albi.hal.science/hal-02050668}

Submitted on 27 Feb 2019

HAL is a multi-disciplinary open access archive for the deposit and dissemination of scientific research documents, whether they are published or not. The documents may come from teaching and research institutions in France or abroad, or from public or private research centers.
L'archive ouverte pluridisciplinaire HAL, est destinée au dépôt et à la diffusion de documents scientifiques de niveau recherche, publiés ou non, émanant des établissements d'enseignement et de recherche français ou étrangers, des laboratoires publics ou privés. 


\title{
Numerical simulation of failure prediction for ceramic tools: comparison with forging test results
}

\author{
F. Deschaux-Beaume ${ }^{\mathrm{a}, *}$, F. Schmidt ${ }^{\mathrm{a}}$, N. Fréty ${ }^{\mathrm{b}}$, T. Cutard $^{\mathrm{a}}$, J.C. Boyer ${ }^{\mathrm{c}}$, C. Levaillant ${ }^{\mathrm{a}}$ \\ ${ }^{\text {a E} E c o l e ~ d e s ~ M i n e s ~ d ' A l b i, ~} 81013$ Albi, France \\ ${ }^{\mathrm{b}}$ Laboratoire de Physico-Chimie de la Matière Condensée, Université Montpellier 2, 34095 Montpellier, France \\ ${ }^{\mathrm{c}}$ INSA-Lyon, 69621 Villeurbanne, France
}

\begin{abstract}
A fracture prediction criterion for brittle materials has been introduced in the POLLUX finite-element code in order to predict the risk-ofrupture of ceramic tools during a forging operation. POLLUX is a software dedicated to the simulation of forging operations, initially developed by INSA (Lyon). The chosen probabilistic fracture model is based on the weakest-link theory and the statistical theory of Weibull. A surface approach or a volume approach can be retained on the basis of the type of critical flaws in the ceramic. Two different criteria are available in order to characterise the stress state, considering the tensile normal stresses and neglecting the compressive stresses. An identification procedure of the critical flaw type is presented for a particular ceramic material. Statistical parameters of ceramic fracture have been determined experimentally using bending tests performed under environmental conditions close to those of forging. A constitutive equation of the workpiece material has been proposed, issued from torsion tests. In order to validate the model in the case of ceramic tools subjected to multi-axial stress states, a particular configuration has been defined to compare the simulation predictions with the experimental results. A forging test has then been developed, in which a billet of superalloy is formed in a ceramic tool up to its fracture at the temperature of $1423 \mathrm{~K}$. The experimental distribution of tool fracture, according to the strain of the billet, is in good agreement with fracture predictions computed by the simulation.
\end{abstract}

Keywords: Finite-element method; Fracture prediction; Ceramics; Forging

\section{Introduction}

Isothermal forging is a very effective forming process for some refractory alloys, such as nickel-based materials. This technique should allow the production of near-net-shape parts directly from preforms of simple shape. However, the process is based on the superplastic properties of the forming materials, and it must be carried out at high temperature and low strain rate. This means that the material for the forging dies, which will be used at the same temperature as the workpiece, must conserve high mechanical properties at high temperature. The forging tools are actually made in Mo-based alloys, but the high reactivity of this element with oxygen imposes the carrying out of the process under an inert gas. The use of ceramic dies should be a less expensive way as the good oxidation resistance of these materials does not require a controlled atmosphere.

However, ceramics are inherently brittle. This characteristic leads, in particular, to a wide variation in the material

\footnotetext{
*Corresponding author. Tel.: +33-563-49-30-00; fax: +33-563-49-30-99.
}

strength. The usual design techniques are, therefore, not suited to ceramic components, and statistics and reliability analysis are needed. Statistical fracture models based on the weakest-link theory have been developed previously. The first probabilistic approach used to account for the scattering in the fracture strength of brittle materials was introduced by Weibull [1]. This model was proposed initially for constant uniaxial stress fields. New criteria were added later to extend the Weibull approach to multi-axial stress fields [2,3]. Another model, developed by Batdorf, combines the weakest-link theory and linear elastic fracture [4].

Except in some simple cases, it is necessary to use numerical simulation in order to predict the risk of failure of ceramic parts with these models. Some studies have been conducted previously in which brittle fracture models were coupled to finite-element simulations in order to calculate the failure probability of particular ceramic structures. In some cases, a post processor has been developed for a specific software to analyse the probabilistic behaviour of simple ceramic structures [5] or of uniaxially-loaded ceramic composites [6]. However, most of the studies in this field 
have been made by the National Aeronautics and Space Administration [7-9]. A computerised design program for the reliability evaluation of ceramic components has been developed from this research work. This software can be coupled to the ANSYS, ABAQUS, NASTRAN and COSMOS commercial finite-element programs. Nevertheless, all of these programs are inconvenient for the simulation of the forging process as large strains take place in the workpiece.

The main objective of this study is to develop a finiteelement model for the failure prediction of ceramic forging dies as a function of the service conditions in order to improve the design of these components.

\section{Description of the failure prediction software}

\subsection{Brittle fracture modelling}

The wide variation in the strength measured for brittle materials is due to the nature and distribution of intrinsic microscopic flaws. These flaws are unavoidably present as a result of the material manufacturing and machining operations, or of a damage process. Distinction must be made between volume and surface flaws, which lead to different strength distributions. In the case of a coarse material microstructure, the volume flaws are generally more critical than the surface flaws. However, the surface flaws may be more critical, for example, in the case of rough machining or of oxidised surface states. The identification of the nature of critical flaws allows one to choose the approach to the analysis. If a surface flaw is chosen, the volume flaws are assumed to be not responsible for the fracture.

As the flaw distribution cannot be determined, some statistical functions are used to describe the strength variability of a component. The Weibull model has been chosen in the present study because the statistical parameters are easy to determine experimentally. The Weibull theory associates a strength $\sigma_{\mathrm{c}}$ to each elementary volume Ve (or elementary surface Se for the surface approach). The strength of the material is characterised by the distribution function of $\sigma_{\mathrm{c}}$, denoted $F(\sigma)$, which is defined as the number of all elementary volumes (surfaces) having $\sigma_{\mathrm{c}} \leq \sigma$, divided by the total volume (external surface). This function also gives the probability $P$ of randomly choosing an elementary volume (surface) having a strength equal to or less than $\sigma$, or also the probability that this volume fails under the stress $\sigma$ :

$P\left(\sigma_{\mathrm{c}} \leq \sigma\right)=F(\sigma)$

Any distribution function may be written in the form

$F(\sigma)=1-\exp (-\phi(\sigma))$

where $\phi(\sigma)$ must be a positive and non-decreasing function, vanishing at a value $\sigma_{\mathrm{u}}$, called the threshold stress.

The advantage of this equation appears when considering the failure probability of the structure constituted by all the elementary volumes (surfaces), using the weakest-link theory. When supposing that the total volume $V$ (surface $S$ ) fails if any one of its parts fails, then the probability of non-failure $P_{\mathrm{NF}}$, under a uniform uniaxial load $\sigma$, is equal to

$P_{\mathrm{NF}}=(1-P)^{n}=\exp (-n \phi(\sigma))$

where $n$ is the number of elementary volumes (surfaces) of the structure $(V=n \mathrm{Ve}$ or $S=n \mathrm{Se})$.

The failure probability $P_{n}$ of the structure takes then the simple form

$P_{n}=1-\exp (-n \phi(\sigma))$

Weibull chose a power form for the function $\phi(\sigma)$, which is the simplest function satisfying the general conditions described previously:

$\phi(\sigma)=\left(\frac{\sigma-\sigma_{\mathrm{u}}}{\sigma_{0}}\right)^{m}$

where $\sigma_{\mathrm{u}}, \sigma_{0}$ and $m$ are parameters depending on the characteristics of the material.

The threshold stress $\sigma_{\mathrm{u}}$ is usually taken as equal to zero. The two other parameters can then be identified easily by comparison with the results of fracture tests.

In the case of a non-uniform uniaxial load $\sigma$, the failure probability takes the integral form

$P_{\mathrm{v}}=1-\exp \left(-\frac{1}{\mathrm{Ve}} \int_{V}\left(\frac{\sigma}{\sigma_{0}}\right)^{m} \mathrm{~d} V\right)$

for the volume approach, or

$P_{\mathrm{s}}=1-\exp \left(-\frac{1}{\mathrm{Se}} \int_{S}\left(\frac{\sigma}{\sigma_{0}}\right)^{m} \mathrm{~d} S\right)$

for the surface approach.

The previous relationship can be generalised to multiaxial stress states. Two strategies are available: the first consists of considering an equivalent stress that characterises the loading at each point, this criteria sometimes leading to unsafe estimates of the failure probability; whilst the second consists of assuming the independence of the actions of each principal tensile stress [2].

Taking into account the first approach, Weibull proposed the calculation of an equivalent stress by averaging the normal tensile stresses in all directions. This model leads to a complex integral formulation, which may increase the computation time.

In order to obtain shorter computation times, only the maximal normal stress has been considered in the present model, as has been done by other investigators [5]. This variable is also the maximal principal stress at each point of the structure.

In the second approach, the fracture probability depends on all the tensile principal stresses. The independence of the actions of the principal stresses means that the reliability of 
the structure is the product of the reliability of the individual principal stresses acting independently. The fracture probability is then given by the following relationship:

$P_{\mathrm{v}}=1-\exp \left(-\frac{1}{\mathrm{Ve}} \int_{V}\left(\left(\frac{\sigma_{1}}{\sigma_{0}}\right)^{m}+\left(\frac{\sigma_{2}}{\sigma_{0}}\right)^{m}+\left(\frac{\sigma_{3}}{\sigma_{0}}\right)^{m}\right) \mathrm{d} V\right)$

for the volume approach, or

$P_{\mathrm{s}}=1-\exp \left(-\frac{1}{\operatorname{Se}} \int_{S}\left(\left(\frac{\sigma_{1}}{\sigma_{0}}\right)^{m}+\left(\frac{\sigma_{2}}{\sigma_{0}}\right)^{m}+\left(\frac{\sigma_{3}}{\sigma_{0}}\right)^{m}\right) \mathrm{d} S\right)$

for the surface approach, where $\sigma_{1}, \sigma_{2}$ and $\sigma_{3}$ are the positive principal stresses or take the value zero for the compressive principal stresses.

It is interesting to note that in both models, the compressive stresses are not taken into account. The fracture is supposed to be always the result of tensile stresses. This choice has been made because the compressive fracture strength of ceramics is three or four times greater than the tensile strength. It is very unlikely that a structure fails under the effect of compressive stresses.

\subsection{Numerical introduction of the model}

The model described previously has been introduced as a subroutine in the POLLUX finite-element code [10]. POLLUX is a specialised software developed by INSA-Lyon [11] in order to predict the stress state in the workpiece and the surrounding tools during a forging process. First, the thermo-mechanical analysis is achieved, leading to the calculation of the stress tensor at each point of the tools, which are supposed to show a perfectly elastic behaviour. Then, the failure probabilities of the tools are determined, using the Weibull parameters and the criteria chosen by the user.

If $\mathrm{Ve}$ or Se are arbitrarily taken to be equal to 1 , the discrete decomposition of the models gives

$\left.P_{\mathrm{v}}=1-\exp -\sum_{\text {iel }} \sum_{\text {ig }}\left(\frac{\sigma_{1}}{\sigma_{0}}\right)^{m} V(\mathrm{ig})\right)$

for the volume approach, or

$P_{\mathrm{s}}=1-\exp -\sum_{\text {inc }}\left(\frac{\sigma_{1}}{\sigma_{0}}\right)^{m} S($ inc $\left.)\right)$

for the surface approach if the maximal principal stress criteria (MPS) is chosen, and

$P_{\mathrm{v}}=1-\exp \left(-\sum_{\mathrm{inc}} \sum_{\mathrm{ig}} \sum_{i=1}^{3}\left(\frac{\sigma_{1}}{\sigma_{0}}\right)^{m} V(\mathrm{ig})\right)$ for the volume approach, or

$P_{\mathrm{s}}=1-\exp -\sum_{\text {inc }} \sum_{i=1}^{3}\left(\frac{\sigma_{i}}{\sigma_{0}}\right)^{m} S($ inc $\left.)\right)$

for the surface approach if the principle of the independent actions (PIA) is chosen.

$\sigma_{1}, \sigma_{2}, \sigma_{3}$ are the principal stresses in the Gauss point ig of element iel or in the contour node inc $\left(\sigma_{1} \geq \sigma_{2} \geq \sigma_{3}\right)$ or are equal to zero for the compressive principal stress. $V(\mathrm{ig})$ is the volume associated with the Gauss point ig, and $S$ (inc) is the surface associated with the contour node inc.

The elementary failure probability DPr, or local risk-ofrupture, is also calculated at each Gauss point in the case of the volume approach, and interpolated to the nodes. This variable corresponds to the fracture probability of an elementary volume of constant size equal to 1 , subjected to the stress state of the point considered. The values can be stored in a data file for a graphical display of the critical regions. Another data file, containing the failure probability of the overall structure as a function of the time or tool displacement, is also generated. At the end of each increment, the program compares the failure probability of the tools to the maximal permissible probability chosen by the user. The computation is interrupted when this limit or the maximal tool course is achieved.

\section{Reliability analysis procedure for ceramic forging tools}

\subsection{Identification of critical flaw type}

Reliability analysis of ceramic structures with the software described previously first requires a failure criteria to be chosen corresponding to the material and the use conditions. If the choice between MPS and PIA criteria cannot be made by considering physical observations, the choice of the type of approach (surface or volume) must be based on these observations.

In the case of a forging process with ceramic tools, the material strength is modified because of the damage inherent to the use conditions. Two types of flaws can be created: surface flaws, essentially produced by the oxidation of the material at the forging temperature; and internal flaws (microcracks), which can be formed because of the mechanical loading of the tool or because of the thermal cycles.

In order to identify the critical flaw type formed during the forging process, the fracture strength of the initial material and the material subjected to a damaging factor (oxidation, mechanical or thermal cycles) are compared. The failure probability of the material, as a function of the stress, is determined using four-point bending tests (see Section 3.2) at room temperature. Fig. 1 shows the experimental points and the corresponding interpolated Weibull functions for the different states of the material. 


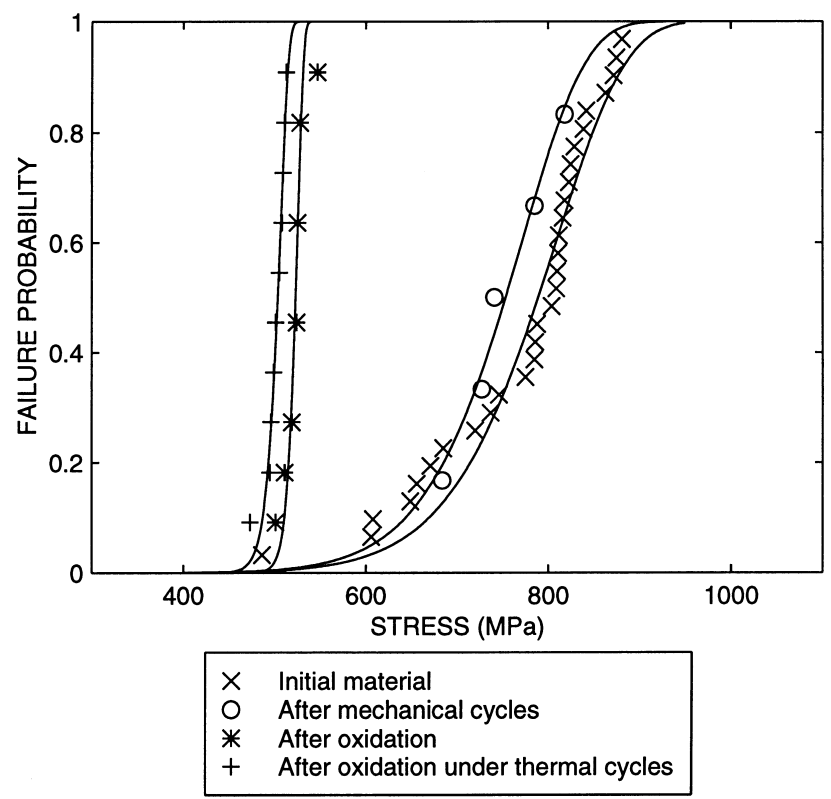

Fig. 1. Fracture strength distribution of the initial and the damaged material.

First, it is to be noted that the oxidised material has a lower strength than the initial material. Then it appears that mechanical and thermal cycles have no significant effects on the material characteristics. The strength distribution of the material after mechanical cycles is, indeed, very close to that of the initial material, and the strength distribution of the material after oxidation and thermal cycles is approximately the same as for the uncycled oxidised material.

This means that the surface flaws, created by the oxidation of the material, are more critical than the initial flaws, or the internal flaws resulting from cyclic loading. According to this result, the surface approach must be chosen for the calculation of the reliability of the ceramic tools during the forging process.

\subsection{Determination of statistical parameters}

The second step of the reliability analysis of the ceramic tool consists of the experimental determination of the statistical parameters of the Weibull model corresponding to the material and the processing conditions. Four-point bending tests (Fig. 2) are carried out at $1423 \mathrm{~K}$ on a set of $N$ specimens oxidised previously at $1423 \mathrm{~K}$ for $50 \mathrm{~h}$. The failure probability of each sample is given by the equation

$P_{\mathrm{ex}}=\frac{i}{N+1}$

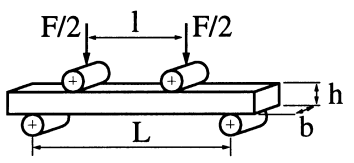

Fig. 2. Four-point bending tests. where $i$ is the rank of the maximal stress $\sigma_{\max }$ in the corresponding specimen at the fracture point. $\sigma_{\max }$ is given by

$\sigma_{\max }=\frac{3}{2} \frac{F(L-1)}{b h^{2}}$

The Weibull distribution corresponding to the bending tests, in the case of surface critical flaws, can be expressed as follows:

$P_{\text {th }}=1-\exp \left(-S_{\text {eff }}\left(\frac{\sigma_{\text {max }}}{\sigma_{\mathrm{u}}}\right)^{m}\right)$

where $S_{\text {eff }}$ is the effective surface determined from the surface in tension, $S$, of a specimen:

$S_{\text {eff }}=\int_{S}\left(\frac{\sigma}{\sigma_{\max }}\right)^{m} \mathrm{~d} S$

Eq. (16) can also be written as

$\ln \left(\ln \left(\frac{1}{1-P_{\mathrm{th}}}\right)\right)=\ln \left(\frac{S_{\mathrm{eff}}}{\sigma_{\mathrm{u}}^{m}}\right)+m \ln \left(\sigma_{\mathrm{max}}\right)$

The Weibull plot, which expresses the evolution of $\ln (\ln (1 /(1-P)))$ as a function of $\ln \left(\sigma_{\max }\right)$, is usually used to represent the experimental results. The Weibull parameters are estimated by fitting Eq. (18) in the experimental points of a Weibull plot, using the least-squares method (Fig. 3). The Weibull parameters $m$ and $\sigma_{\mathrm{u}}$ obtained are equal to 12.4 and $580 \mathrm{MPa}$, respectively.

\subsection{Determination of the workpiece rheology}

A further set of data required for the reliability analysis is the rheological behaviour of the workpiece material. Under forging conditions, the superalloys have a superplastic behaviour. In consequence, the flow stress depends on the strain rate. In order to determine the constitutive equation of

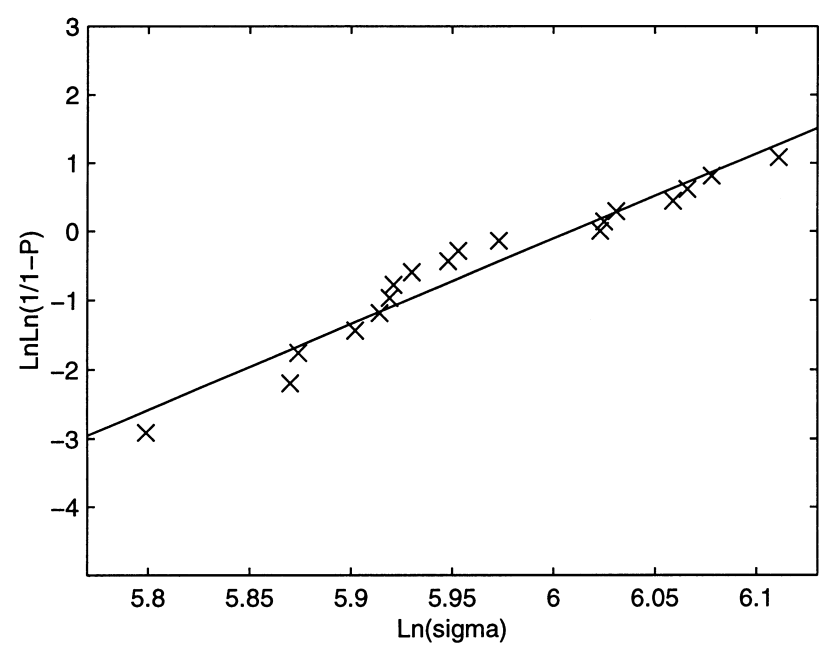

Fig. 3. Weibull's plot of the four-point bending strength at $1423 \mathrm{~K}$ of ceramic specimens oxidised previously at $1423 \mathrm{~K}$ for $50 \mathrm{~h}$. 
this material, torsion tests have been carried out at the forging temperature with three different strain rates ranging from 0.03 to $0.3 \mathrm{~s}^{-1}$. During the test, the experimental torque is measured as a function of the turn number $T$. The equivalent strain rate $\dot{\bar{\varepsilon}}$ and strain $\bar{\varepsilon}$, calculated at the periphery of the specimen, are given by

$\dot{\bar{\varepsilon}}=2 \pi \frac{\dot{T} R}{\sqrt{3} b} \quad$ with $\quad \dot{T}=\frac{\mathrm{d} T}{\mathrm{~d} t}$

$\bar{\varepsilon}=2 \pi \frac{T R}{\sqrt{3} b}$

where $R$ and $b$ are the radius and the length, respectively, of the specimen. The Von Mises equivalent stress $\bar{\sigma}$ is given by

$\bar{\sigma}=\frac{\sqrt{3}}{2 \pi R}(3+\tilde{n}+\tilde{m})$

where $\tilde{n}$ and $\tilde{m}$ represent the sensitivity of the torque to the strain and to the strain rate.

The evolution of $\bar{\sigma}$ with $\bar{\varepsilon}$ is plotted in Fig. 4 for the different strain rates. The curves show a stress peak $\left(\bar{\sigma}_{\mathrm{p}}\right)$, followed by softening and stabilisation of the stress values $\left(\bar{\sigma}_{\mathrm{s}}\right)$.

According to Berdin et al. [12], the stress peak observed in this type of material at low strain levels is due to a dynamic recrystallisation phenomenon. The material behaviour can be approximated by the model

$\bar{\sigma}=(1-\alpha) \bar{\sigma}_{\mathrm{p}}+\alpha \bar{\sigma}_{\mathrm{s}}$

with

$\bar{\sigma}_{\mathrm{p}}=A_{\mathrm{p}} \dot{\bar{\varepsilon}}^{\mathrm{mp}}, \bar{\sigma}_{\mathrm{s}}=A_{\mathrm{s}} \dot{\bar{\varepsilon}}^{\mathrm{ms}}$

where the different constants are determined by fitting the model to the experimental results. $\mathrm{mp}$ and $\mathrm{ms}$ are the strain rate sensitivity coefficients, equal to 0.22 and 0.15 , respectively, whilst $A_{\mathrm{p}}$ and $A_{\mathrm{s}}$ depend only on the temperature and are equal to 150.1 and 109.6 , respectively. $\alpha$ represents

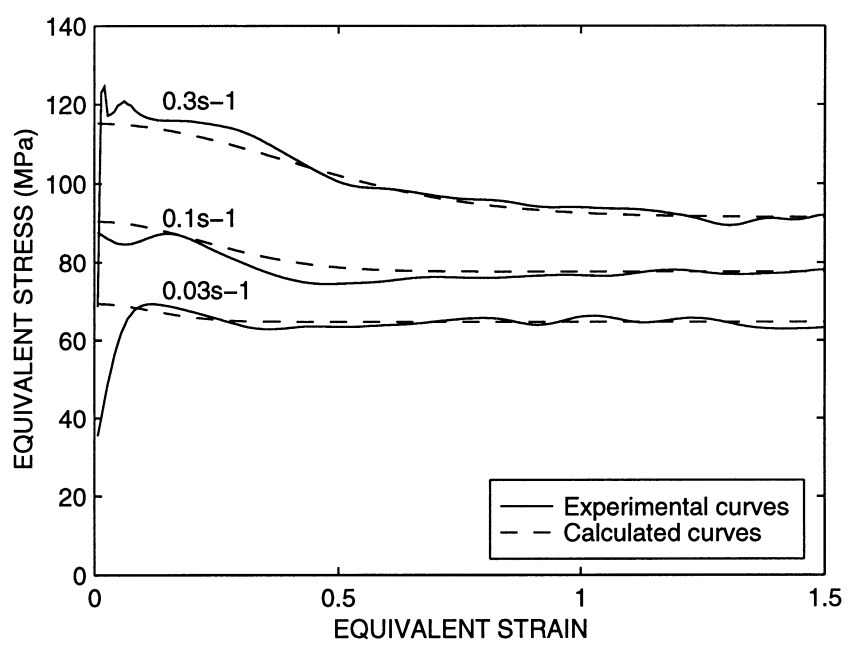

Fig. 4. Stress-strain curves obtained from torsion test at $1423 \mathrm{~K}$ and calculated from the model. the recrystallized fraction, given by an Avrami type equation [12]. Fig. 4 shows a good agreement between the experimental results and the curves calculated from this model.

\section{Confrontation with the forging-fracture test results}

\subsection{Description of the forging test}

In order to validate the model and the approach described previously, fracture tests have been carried out on ceramic tools under near-forging conditions, the results being compared to the numerical results of the tool reliability. Fig. 5 shows the samples chosen for this confrontation. The ceramic and the workpiece are heated to $1423 \mathrm{~K}$ in an induction furnace. During the test under isothermal conditions, a superalloy workpiece is pressed by a punch into the ceramic specimen until its fracture. The forging load and the punch displacement are measured throughout the tests.

After the tests, realised under the same conditions on a set of ceramic specimens, the failure probability calculated from Eq. (14) is expressed as a function of the punch displacement. A program of 10 fracture tests has been planned to obtain an experimental distribution of ceramic fracture.

\subsection{Failure probability calculation}

Good agreement between the experimental results and the numerical simulation predictions has been observed. First, the calculation of the stress state in the specimen has shown that the orthoradial stress is the maximal component of the stress tensor in the ceramic (Fig. 6). This predicts that, at fracture, the cracks in the ceramic specimen must be longitudinal: this has been verified experimentally and can be observed clearly in Fig. 5. Secondly, the failure distribution of the ceramic specimens, expressed as a function of the punch displacement, obtained from the numerical calculation are presented in Fig. 7. The experimental values measured during the forging tests are very close to the calculated values.

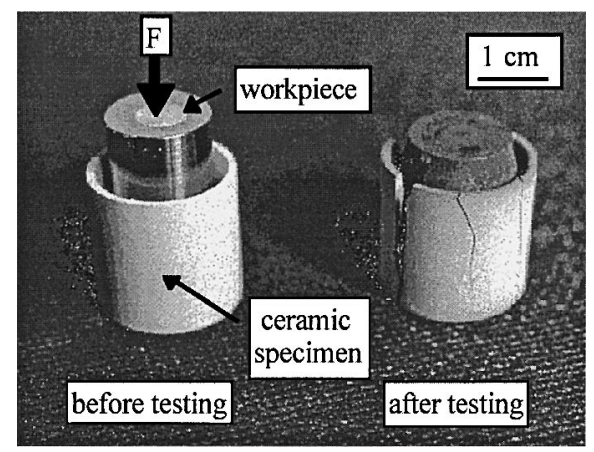

Fig. 5. Ceramic specimens and the workpiece used for the fracture tests. 


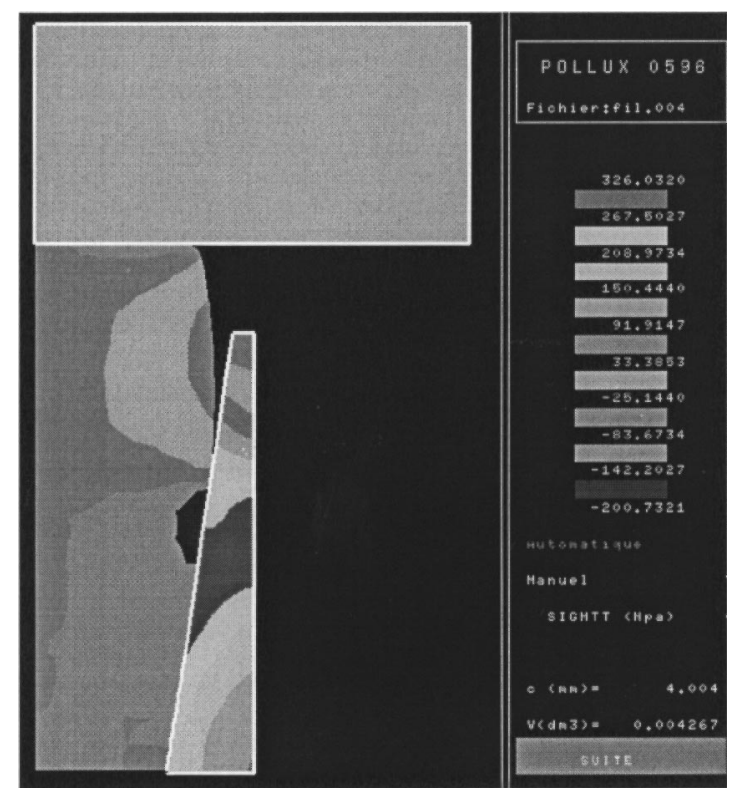

Fig. 6. Orthoradial stress distribution obtained from the forging test simulation after a punch displacement of $4 \mathrm{~mm}$.

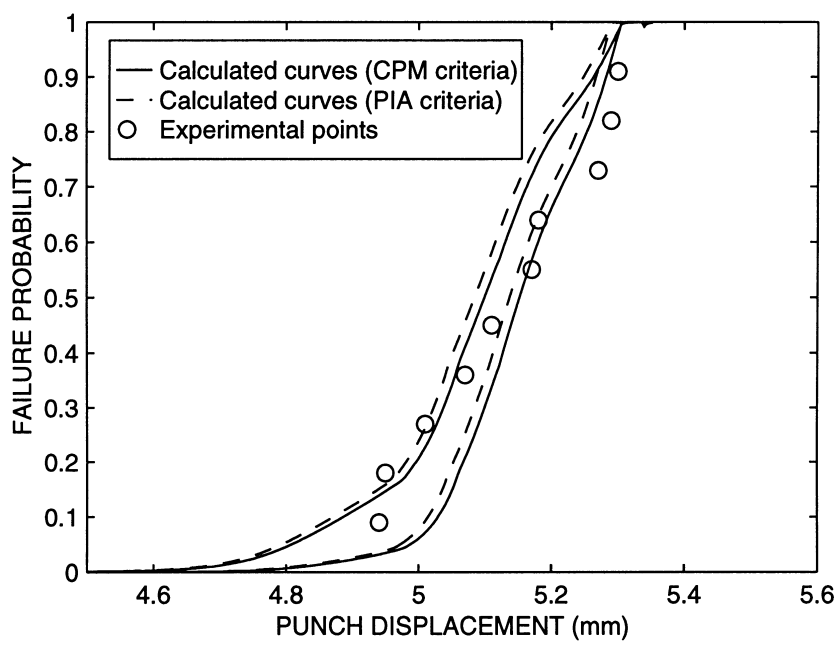

Fig. 7. Experimental and calculated failure probability of the ceramic specimen.

Both MPS and PIA criteria available for the calculation have been tested in order to determine as to which is more efficient for the present case. Nevertheless, the choice seems to be difficult because of the weak difference obtained from the two criteria. This is so because, for the ceramic geometry chosen, one of the principal stresses is always predominant, and the other two have no significant effects on the risk-ofrupture. Thus, except for quasi-equi-biaxial stress states, both the MPS and the PIA criteria are efficient in predicting the failure probability of the ceramic material chosen.

\section{Conclusions}

A fracture-prediction program for brittle materials has been introduced in the finite-element code POLLUX in order to predict the risk-of-rupture of ceramic tools during high temperature forging operations. For efficient use, the following characteristics must be known: (i) the type of the critical flaws in the ceramic; (ii) the statistical parameters of the fracture strength distribution of the ceramic; and (iii) the metal workpiece rheology. These characteristics can be measured by classical mechanical tests (bending, torsion or tension tests).

The software is then a very useful prediction tool for the fracture of ceramic parts as: (i) it permits the prediction of the failure probability of a ceramic tool with a given geometry, as a function of its use conditions; and (ii) it can also give elements to predict the localisation and the orientation of the fracture cracks. So, such a software can be very helpful in the design of the geometry and of the dimension of large ceramic tools.

The comparison between the numerical simulation results and the forging fracture tests has shown a good agreement. Nevertheless, the test configuration has not permitted differentiation between the two available criteria (MPS and PIA). Complementary experimental tests could be performed in order to have a more complete comparison, particularly for a quasi-equi-biaxial stress state. Other failure criteria could also be considered, for example, taking into account the shear stresses in the ceramic. Future developments of the software forecast the introduction of this type of criteria.

\section{References}

[1] W.A. Weibull, J. Appl. Mech. 18(3) (1951) 293.

[2] A.M. Freudenthal, in: H. Liebowitz (Ed.), Fracture, vol. 2, Academic Press, New York, 1968.

[3] A.G. Evans, J. Am. Ceram. Soc. 61 (1978) 302.

[4] S.B. Batdorf, J.G. Crose, J. Appl. Mech. 41(2) (1974) 459.

[5] C. Berdin, D. Baptiste, D. Jeulin, G. Cailletaud, in: Proc. of Fracture Processes in Brittle Materials, Noordwijk, Holland, June 1991.

[6] L. Guillaumat, J. Lamon, in: Proc. of Journée nationales sur les composites, Saint-Etienne, November 22, 1994.

[7] J.P. Gyekenyesi et al., J. Eng. Gas Turbines Power 108 (1986) 540.

[8] J.P. Gyekenyesi, N.N. Nemeth, J. Eng. Gas Turbines Power 109 (1987) 274.

[9] N.N. Nemeth, L.M. Powers, L.A. Janosik, J.P. Gyekenyesi, Am. Ceram. Soc. Bull. 72(12) (1993) 59.

[10] F. Deschaux-Beaume, F. Schmidt, N. Frety, J.C. Boyer, C. Levaillant, J. Mater. Proc. Technol. 3 (1998) 100.

[11] B. Michel, J.C. Boyer, Adv. Mater. Processing Technol. 3 (1993) 1701.

[12] M. Soucail, L. Naze, A. Moal, J.P. Ferte, Revue Scientifique Snecma 4 (1993) 33. 